\title{
Evaluation of alternative criteria for determining the optimal location of RWIS stations
}

\author{
Tae J. Kwon · Liping Fu
}

Received: 3 February 2013/Revised: 15 February 2013/Accepted: 18 February 2013/Published online: 6 June 2013

(C) The Author(s) 2013. This article is published with open access at Springerlink.com

\begin{abstract}
This article presents a comprehensive framework for determining the location of road weather information system (RWIS) stations over a regional road network. In the proposed methodology, the region is divided into a grid of equal-sized zones which are considered as the minimum spatial unit for allocating a candidate set of RWIS stations. These zones are ranked according to a set of pre-specified criteria that reflect the needs for, and potential benefits from, real-time road weather information, including road surface temperature variability, precipitation, network traffic, and collision patterns. A case study based on the existing RWIS network in the province of Ontario was conducted to illustrate the major features of the proposed method and evaluate the implications of alternative location selection criteria. The findings of the study suggest that it is feasible to develop a systematic process for locating RWIS stations using an integrated location criterion to capture multiple factors being considered in practice. The study has also revealed the need to establish quantitative models for estimating the benefit of real-time information from RWIS stations, which is the foundation of a cost-benefit-based RWIS location optimization model.
\end{abstract}

T. J. Kwon $(\bowtie) \cdot$ L. Fu

Department of Civil and Environmental Engineering,

University of Waterloo, 200 University Ave West,

Waterloo, ON N2L 3G1, Canada

e-mail: tkwon@uwaterloo.ca

L. Fu

e-mail: 1fu@uwaterloo.ca

L. Fu

School of Transportation and Logistics, Southwest Jiaotong

University, Chengdu, China
Keywords RWIS - Road surface temperature - Accident rate Traffic volume Location selection criteria

\section{Introduction}

Accurate and timely information on road weather and surface conditions (RSC) in winter seasons is a necessity for road authorities to optimize their winter maintenance operations and improve the safety and mobility of the traveling public. One of the primary tools for acquiring this information is a road weather information system (RWIS). The term RWIS is generally used to describe a system consisting of many environmental sensors placed at the roadside or in the roadway, connected together for collecting real-time local atmospheric and pavement conditions information [1]. Data collected by these series of sensors are subsequently examined and/or manipulated, and utilized in generating site-specific road weather and condition forecasts. With such information, hazardous highway segments are flagged to alert maintenance personnel for prompt maintenance operations, which in turn could effectively reduce salt usage and, thus, detrimental effect on the environment [2]. Furthermore, RWIS is essential for some innovative snow- and ice-control strategies such as direct liquid application (DLA, i.e., de-icing and anti-icing), and fixed automated spray technology (FAST) [3]. More importantly, some prior studies have shown that localized road weather and condition information from RWIS can produce direct benefits of reduced costs because of, for instance, efficient use of labor, materials, and equipment for WRM operations [4]. Moreover, there are also some indirect benefits of using RWIS information. For instance, motorists can save their travel time and reduce fuel consumption from the enhanced 
traffic signal timing, and can also be warned of real-time hazards from dynamic warning systems [5].

Acknowledging these potential and perceived benefits of RWIS, many transportation agencies have invested millions of dollars in establishing their current RWIS network and continue expanding their network for better winter maintenance decision support and traveler information provision. While effective in providing real-time information on road weather and surface conditions, RWIS stations are expensive to install and operate, and therefore can only be deployed at a limited number of locations. Considering the vast road network varied conditions of which need to be constantly monitored locally and regionally during winter weather events, RWIS stations must be placed strategically so that they can collectively provide informative inputs required for accurate estimation of the road weather and surface conditions of the whole highway network.

There are a few studies conducted previously, which have attempted to determine the location of RWIS stations at the regional level. Eriksson and Norrman [6] undertook a study on optimally locating RWIS stations in Sweden where they identified conditions hazardous to road transport as a criterion for locating RWIS stations. In their study, they identified 10 different slipperiness types using one winter season of RWIS data, and linearly regressed each type with location attributes including latitude, longitude, elevation, distance to coast, etc. With the resulting regression model, they mapped out the occurrences of each slipperiness type over the entire study area. Candidate RWIS sites were recommended based on the estimated slipperiness counts and four different landuse groups. Although the proposed method in that study seems to provide a good reference for analysis of station locations with respect to various locational attributes and landuse types, it is a heuristic approach considering only one location criterion-road weather condition. In addition, those authors did not provide much explanations/justifications as to how their four landuse groups such as forest/water, open/water, forest, and open areas were determined. Such categorization schemes can be deemed subjective and thus scientifically less persuasive.

World Weather Watch [7] conducted a study involving a climatological analysis for the purpose of optimizing the RWIS station locations. The study extensively reviewed micrometeorological variations by investigating local physiography, topography, temperature, and snowfall precipitation amount within the study area. The study also took into account the hotspots identified by the maintenance personnel which require regular monitoring. By combining all those factors, a list of high-risk sites are identified as the recommended locations for new RWIS stations in the region. A similar yet more inclusive study was carried out by Alberta Department of Transportation in collaboration with AECOM and Telvent, in which they proposed a sound approach to determine the location and density of RWIS stations, by identifying and analyzing the RWIS-deficient regions (RDR), and by following general budget guidelines, respectively [8]. Their approach consists of two components namely macro and micro assessments. In the macro-assessment phase, the authors took several factors into account when determining the RDR such as traffic loads, accident rates, climatic zones, availability of meteorological information, and discussions with regional road maintenance personnel, and key stakeholders. In the micro-assessment phase, among the selected subset of potential new RWIS locations, a final site was selected by conducting detailed field visits to ensure their suitability and feasibility (e.g., appropriate sensor selection/configuration, budget, access to power, etc.) for installing a new RWIS station.

These past studies, while logical in methodology, lack scientific and systematic formulation of justification on how all those factors/criteria are put together to determine the potential high-risk sites. More importantly, both studies had failed to provide a clear linkage between the considered criteria and the purposes of RWIS stations. For instance, the latter study included accident rates as one of their potential location selection criteria, but did not establish a solid rationale as to why such a criterion should be given a priority when choosing a new location. In other words, what is the relationship between the accident rate and the availability of a RWIS station? Without a valid justification/explanation on why each location selection criterion is considered and utilized, incorporation of such selection criteria in the studies cannot be legitimized.

Currently, majority of provincial and municipal transportation agencies do not have a systematic approach for determining the suitable RWIS locations, but rather rely heavily on the experience of regional/local maintenance personnel. According to the survey conducted for synthesizing the current best practices on how RWIS stations are deployed, most respondents claimed to consider different weather zones, which are the areas with historically heavy snow, high wind, low-lying road segments, etc. As for the non-weather-related factors, they would mainly consider collision rate, traffic loads, frequency of WRM operations, and road class. All the information is put together through a series of face-to-face meetings with key stakeholders and field experts to narrow down various candidate locations to a manageable size and decide based on the budget availability. Finding a resolution through this process is laborious and time-consuming one thereby requiring greater manpower.

As described above, past efforts and current practices for determining the location of RWIS stations have several limitations. In particular, what constitutes a good RWIS location? What location selection criteria should be 
included in the analysis for determining the potential RWIS stations? What are the pros and cons of these criteria used for locating RWIS stations? How easily can the RWIS station location scheme be applied, adopted, and utilized by the RWIS network expansion planners? These questions represent the main concern of this study.

The main objective of this study is to construct a systematic approach/framework for determining the candidate RWIS station location. The article is organized as follows. The first section presents an introduction to the topic under review. The second section discusses several criteria for identifying a "good" location of RWIS stations that are also used as a basis of location-ranking criteria in this study. The third section describes the proposed methodology to be undertaken in this study. Analysis and evaluation of considered criteria using a case study based on Ontario RWIS network are presented in the fourth section. The last section is composed of conclusions and recommendations for future study.

\section{Regional location selection criteria}

It is critical to clearly define the criteria that can be used to measure the "goodness" of a location for installing a RWIS station. To identify the criteria, we must start with determining the values or benefits of RWIS stations. As discussed previously, RWIS stations are installed to collect real-time RSC data, and their values are reflected in the use of these RWIS data, including improved mobility and safety (i.e., beneficial for motorists), and reduced winter road maintenance (WRM) costs and salt usage (i.e., beneficial for agency and environment). These benefits are the basis for the criteria defined in the following section.

\subsection{Weather-related factors}

Intuitively, RWIS stations should be installed in areas that experience severe yet, less-predictable weather patterns, which thus need real-time monitoring the most. Therefore, it is important to analyze the spatial distribution patterns of some critical weather variables such as temperature and precipitation. For example, the variability of surface temperature (VST) and mean surface temperature (MST) are important factors to be considered as they can provide a definite measure of how much the surface temperature would vary over time and space. Late November to early December is the time of year with the highest probabilities of having black-ice or frost. The higher elevation and greater distance away from large water bodies can both contribute to generating colder surface temperatures. This gradually leads to a period of longer winter months with higher likelihood of having frost on road surface, and thus exposes motorists to great danger.

Snowfall amount is another important factor as it makes logical sense that a RWIS station needs to be situated in areas where snowfalls occur frequently. This is particularly true when, having a better monitoring capability can intuitively increase mobility and safety by performing a more prompt WRM operation [4]. Hence, these three weather factors are included in the analysis for selecting a good location of a RWIS station.

\subsection{Traffic-related factors}

The benefits received by RWIS can intuitively increase by placing them in locations where a greater number of traveling publics can be served. For this reason, many transportation agencies are inclined to incorporate macro-level traffic criteria such as collisions, traffic volume, and highway type (HT) [1, 2]. A recent study conducted by Greening et al. [9] showed that a well-maintained RWIS network would in fact reduce the accident rates by a significant amount, which in turn would bring huge savings.

Notwithstanding the fact that they ignored other confounding factors such as a general vehicle technology advancement, which could also have led to the reduction of accident rates, their study clearly demonstrated that the use of RWIS information could potentially prevent accidents by prompt WRM operations. Furthermore, the survey dedicated to providing the current practices of deploying a RWIS system showed that more than $60 \%$ of DOTs who participated would also consider highway class along with collision rate and traffic volume. Their intension for taking highway class into account is similar to considering traffic volume in the context of providing benefits to more number of road users. As such, the three traffic-related factors mentioned above are included as location selection criteria in this study.

\section{Methodology}

In this section, the methodology undertaken to carry out the analysis is briefly described.

Figure 1 shows a flowchart of the proposed method for analyzing and evaluating different location selection criteria for choosing an ideal RWIS station location. As shown in the figure, the first step is to divide the region under investigation into a grid of equal-sized zones (i.e., cells) which are assumed to be the minimum spatial unit for allocating a candidate set of RWIS locations. Once the grid covering the entire study area is created, then major road segments, including highways and arterials, are superimposed onto the grid in such a way that only the cells 
containing the road segments can be selected for further analysis. This step helps one single out only the candidate locations of RWIS stations from all cells, reducing the degree of complexity by having only the "important" cells in the analysis and evaluation phases. Six different criteria are prepared, processed and/or modeled (a point that will be discussed later in this article), and are then used to assign an averaged unique value to each cell in the grid such that each cell can be readily ranked to determine "more" or "less" important sites. Finally, these ranked location criteria are evaluated using Ontario's current RWIS network.

\section{Case study}

\subsection{Ontario RWIS network}

Ontario Ministry of Transportation (MTO) installed its first RWIS station in 1991 as a research and development project. Over the years, the number of stations has increased, mostly in the southern part of Ontario where the road network is the most dense. Since then, the benefits of RWIS being an effective tool for proactively deploying
WRM operation have been recognized [2]. As such, MTO installed additional 85 stations between 2001 and 2004 in a series of three phases. Phase 1 (25 stations) primarily focused on installing along the key economic corridors (i.e., Hwy 401, 400, and 417), whereas Phases 2 and 3 (60 stations) focused on installing stations in the areas where larger gaps previously existed in both the northern and the southern parts. MTO has been expanding the network each year and currently has a total of 140 RWIS stations as depicted in Fig. 2. Figure 2 shows black pushpins and red lines representing the location of individual RWIS stations and the Ontario highway network, respectively. The figure also shows a grid of equal-sized cells each having an area of $50 \times 50 \mathrm{~km}^{2}$. This spatial resolution was decided based on the survey, which revealed that the most provinces and states would typically keep a distance of $40-50 \mathrm{~km}$ between two stations. However, it is important to note that such a criterion is not a requirement, and can vary according to the needs.

As described earlier, the road network is used as a constraint to extract only the matching cells as the other cells that do not match cannot have a RWIS station (e.g., a cell covering, for instance, the lake is not considered as a potential candidate site).

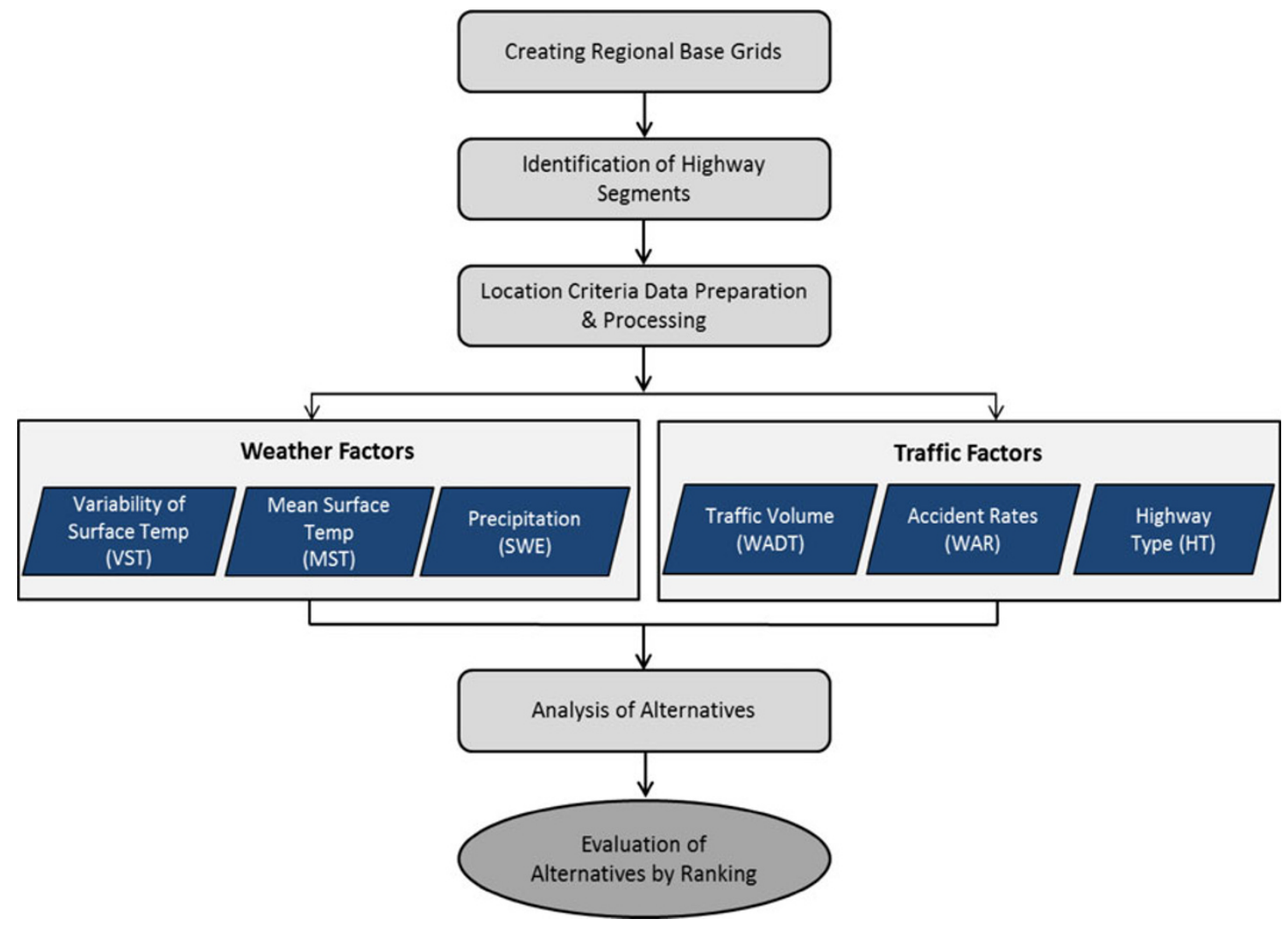

Fig. 1 Flowchart of evaluating alternatives for choosing a RWIS station location 


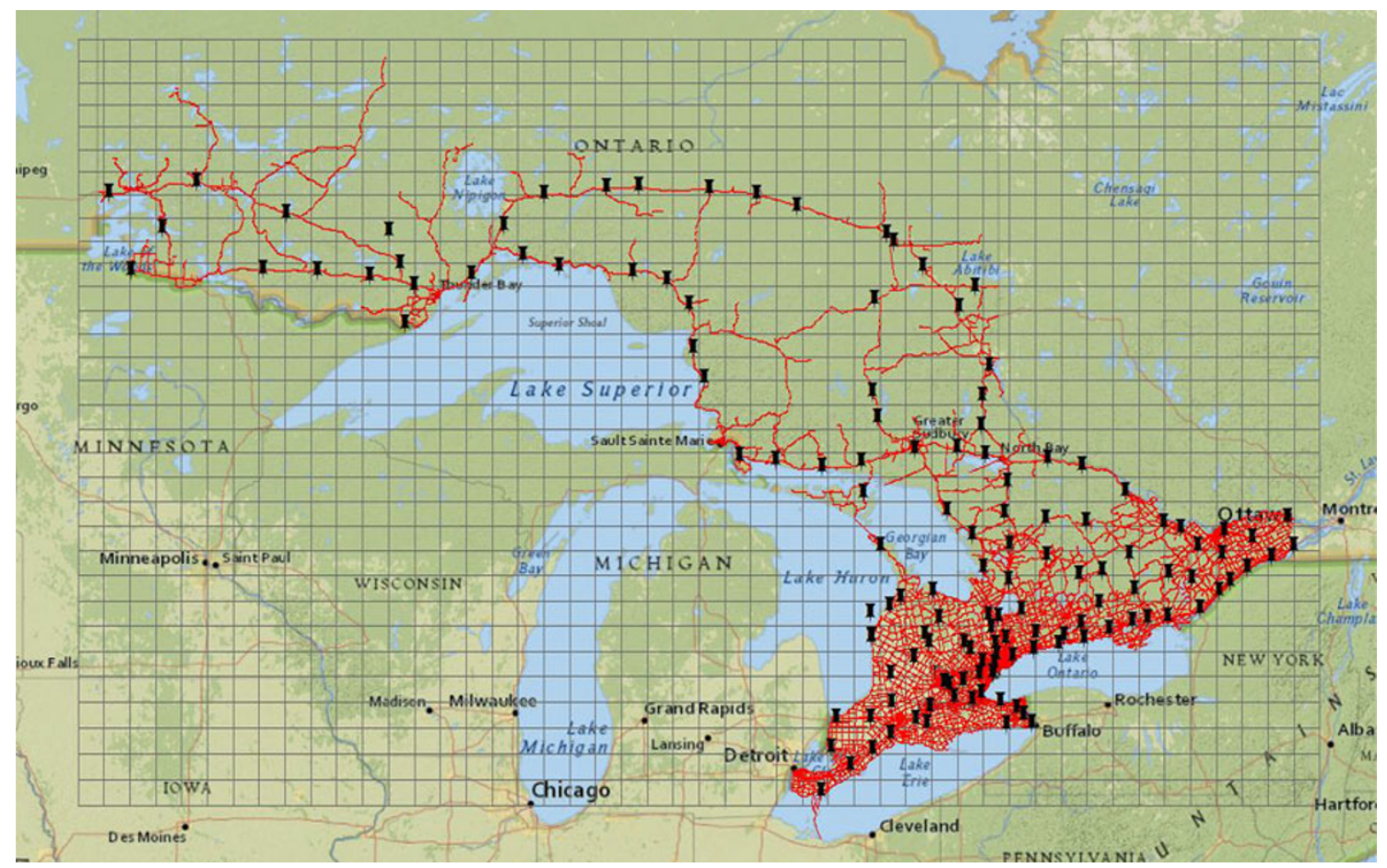

Fig. 2 Current Ontario RWIS Network with 140 stations

4.2 Data preparation and analysis of the location selection criteria

\subsubsection{Modeling of VST and MST}

The two dependent variables of interest are VST and MST, which can be defined by calculating the standard deviation of surface temperature, and the average of minimum surface temperature, respectively, using multiple-year data. The environmental variables (i.e., auxiliary information) used in this study include latitude (lat), longitude (long), elevation (elev), distance from water $\left(d_{w}\right)$, and relative topography $\left(R T_{1}\right.$, $R T_{3}$, and $R T_{5}$ ). The justification for choosing such variables are that latitude is expected to affect the spatial variation of surface temperature, whereas longitude may capture the influence of winds. Elevation in meters above mean sea level could be linked to the VST (e.g., the higher the elevation, lower the temperature), and distance from large water bodies, the Euclidean distance in kilometers, represents the degree of continentality [6]. Finally, relative topography is included to describe the exposure, and is calculated by taking the difference in elevation between each station location and an average of pixels within the respective radius range (e.g., 1, 3, and $5 \mathrm{~km}$ ). Since the monthly variation of surface temperature can vary significantly from one month to another, the two dependent variables, VST and MST, are modeled on a monthly basis. Owing to data unavailability and deficiency, 3-year surface temperature data collected during 2006-2008 from a total of 45 Ontario RWIS stations for the month of
January are used for modeling. ArcGIS 10.1 is used as a base platform for this study, where digital elevation model (DEM) with 1-km spatial resolution as well as water layers including lakes and sea are utilized to obtain the aforementioned auxiliary information. Once all the required information has been obtained, SPSS is used to perform the multiple linear regression (MLR) analysis with all variables being tested at the $5 \%$ significance level. The resulting equations for two dependent variables are as follows:

$$
\begin{aligned}
V S T= & 0.403(\text { lat })+0.076(\text { long })+0.161\left(\text { dis }_{w}\right) \\
& -0.011\left(R T_{5}\right)-5.974 \\
R^{2}= & 72.2 \% \\
M S T= & -2.398(\text { lat })-0.518(\text { long })-0.016(\text { elev }) \\
& +0.296\left(\text { dis }_{w}\right)-0.049\left(R T_{3}\right)+61.937 \\
R^{2}= & 88.3 \%
\end{aligned}
$$

To construct the continuous surface to be used later for evaluating different alternatives, a similar approach is undertaken to extract all the required auxiliary information from all available cells, where the above calibrated equations are used to calculate both VST and MST values for each cell. Figure 3 shows the VST and MST maps. Note that only the cells that contain roads are used for analysis as previously described.

From Eqs. (1) and (2), it can be clearly seen that all regression coefficients make intuitive sense. For instance, latitude, longitude, and distance to water have a positive correlation with VST implying that as values of each 

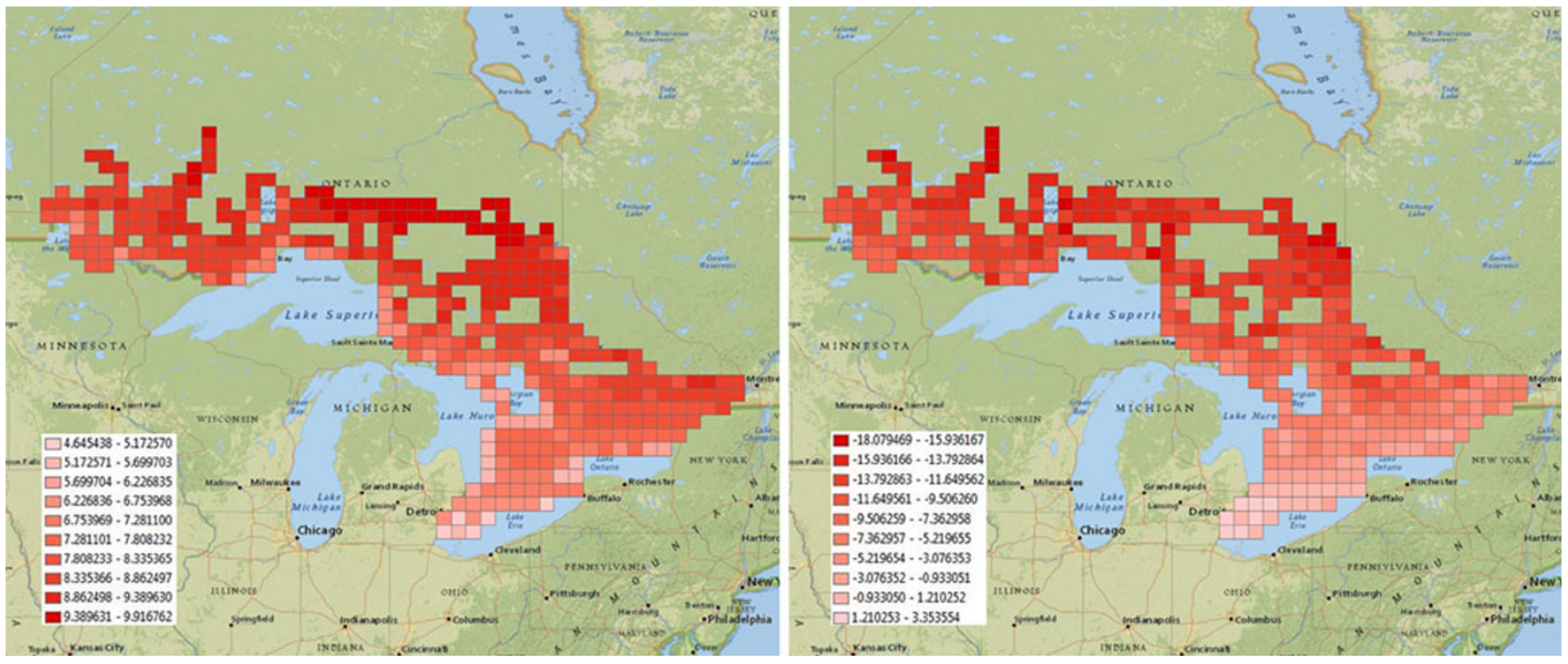

Fig. 3 Processed VST (left) and MST (right) Maps

parameter increase, so does the VST. It is true particularly during colder months that surface temperature would likely vary at a greater range at high latitude regions. Furthermore, VST is likely higher as it moves into deeper continents which are typically more mountainous causing a larger temperature variation. These phenomena are explained graphically in Fig. 3 (left) that VST cells in southern regions and/or nearby lakes tend to exhibit less variation compared with other cells. As for the MST, all the regression coefficients except for distance to water are found to be negative. This is observed since the minimum surface temperature would drop as it moves to the northern regions with higher elevations. Notice that MST can vary by a significant amount $\left(\sim 20{ }^{\circ} \mathrm{C}\right)$ between the northernmost and southernmost cells in Fig. 3 (right). A positive correlation of distance to water represents that as it moves away from water, temperature would likely drop because of the lack of significant water bodies nearby [10].

\subsubsection{Processing and integration of other location selection criteria}

4.2.2.1 Precipitation (snowfall) Distribution of precipitation amounts, particularly of snowfall amounts must thoroughly be investigated to determine the regions where heavy snowfalls are likely to occur so that recommendations for RWIS stations can be made accordingly. This can be done by analyzing the long-term historical snowfall observations. Daymet is an online weather data archive where daily surface weather and climatological summaries are available for public use [11]. Snow-water-equivalent (SWE), which describes the amount of water contained within the snowpack expressed in $\mathrm{kg} / \mathrm{m}^{2}$, is estimated such that biases in shortwave radiation estimates related to multiple reflections between the surface and atmosphere are reduced [12]. The average annual summary maps for SWE are obtained for periods from 2001 and 2005 covering the entire North America. Since these files came in a raster format, 5-year map is generated by taking a sum of all available SWE layers using ArcGIS 10.1. Once all the maps have been combined and averaged, each cell is assigned with the corresponding SWE value (i.e., sum of all SWE data within each cell) for the entire grid. In Fig. 4, it can be seen that central regions seem to have the most snowfalls, and the amounts are being gradually diminished as it moves to outer regions. The farthest southern regions appeared to have the least amount of snow.

\subsubsection{Traffic volume (WADT), accident rate (WAR), and} highway type (HT) It was emphasized earlier that a RWIS station should be located in places where traffic volumes and accident rates are high such that the benefits to road users can be maximized. This reasoning can also be applied to HT where higher classes of highways should be given a priority when installing a RWIS station. For this reason, winter average daily traffic (WADT), winter accident rate (WAR), and HT have been collected and processed to be implemented as RWIS location selection criteria. Traffic information management is centered at the MTO-provided data for the 2000-2010 provincial highways winter traffic volumes and accident numbers along with highway class, all of which are geocoded with linear highway-referencing system (LHRS). LHRS can be defined as a location-referencing method to easily identify a specific location with respect to a known point [13]. MTO currently has a total of 2,588 geocoded locations across the province as shown in Fig. 5a. With these geocoded locations, WADT data have been mapped onto the grid where the data are averaged and 

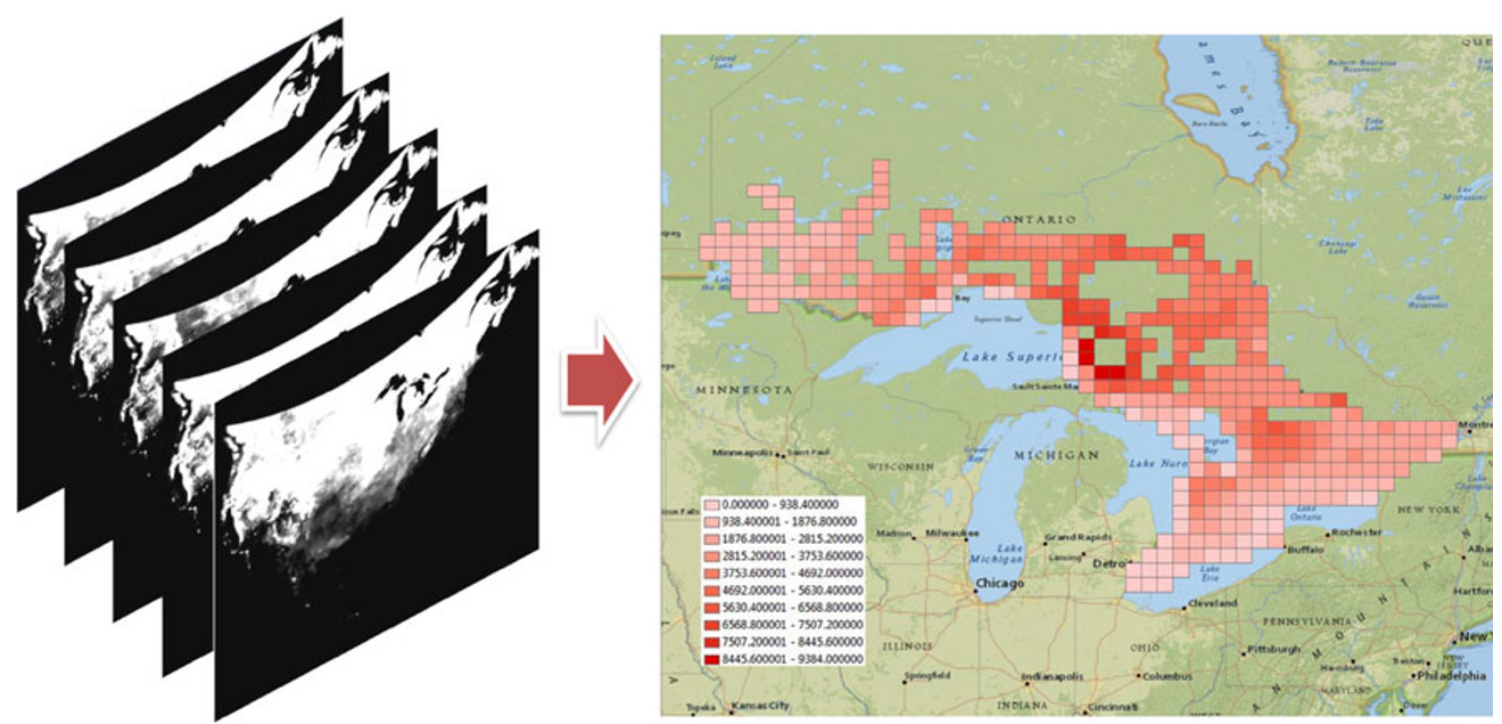

Fig. 4 Five annual average SWE maps (left) and the 5-year Average SWE map (right)

assigned to the corresponding cells. WAR used in the analysis is defined as the number of reportable accidents occurring during winter months on a particular highway section for every million vehicle kilometers (MVK) traveled on that section during the same period, and can be expressed as

$W A R=\frac{\text { Accident Number }(\text { WAN })}{\left(\frac{\text { WADT } \times 212 \times \text { Section Length in } k m)}{1,000,000}\right)}$

Representative section lengths for all geocoded points are used when calculating WAR. As for HT, four different types that are currently being used and have been classified by MTO are used. Following the similar approach of the other traffic dataset, HT data have been first geocoded using the LHRS, and then the averaged values are assigned to each cell. Figure $5 \mathrm{~b}-\mathrm{d}$ depicts the processed WAR, WADT, and HT, respectively. Note that all these three figures are classified by 10 equal intervals. As can be seen in the figure, WAR and WADT data are appeared to share some common traits that there are many number of "high-risk" cells in the southern region for having relatively heavier traffic loads and higher accident rates. This makes a logical sense that increase in exposure would likely increase the number of accidents. On the other hand, northern regions consist of many low-valued cells indicating that they are less important when considering traffic as a location criterion. Similar conclusions can be drawn by analyzing the HT figure that a great number of high-class highways are situated in the southern region suggesting the needs of RWIS stations.

\subsection{Evaluation of alternatives}

In this section, different alternatives are evaluated by applying them to "relocate" Ontario's existing 140 RWIS stations and comparing the results to their current locations. For each alternative, the candidate cells are sorted based on their values of the given selection criterion in a descending order and the top 140 cells are identified accordingly as the new best locations based on that alternative. The percent of cells (locations) matched is calculated as a performance measure.

Before the evaluation, a correlation analysis has been carried out to resolve any possible occurrence of multicollinearity, which could essentially affect and thus produce biased results when two or more factors are added. Spearman's coefficient of rank correlation, denoted as $\rho$ is a nonparametric measure of statistical dependence between any two ranked variables, which are assumed to have a monotonic relationship. Its product ranges between -1 and 1 , indicative of strong negative and positive correlations, respectively. As such, this method was used to assess the correlation with other location selection criteria under analysis. The equation for Spearman's rank correlation coefficient is expressed as [14]

$\rho=1-\frac{6 \sum d_{i}^{2}}{n\left(n^{2}-1\right)}$

where $d_{i}$ is a difference in paired rank; and $n$ is a total number of cases, which in our case is 377 (i.e., a total number of cells in the grid). Table 1 summarizes the Spearman's rank correlation coefficients for all the variables considered in this study.

As anticipated (from a visual inspection of the earlier figures), VST and MST, and WAR and WADT are found to be moderately correlated. Such findings are reasonable since, for example, the rate of accident would vastly be influenced by the number of travelers, or vice versa. The 
(a)

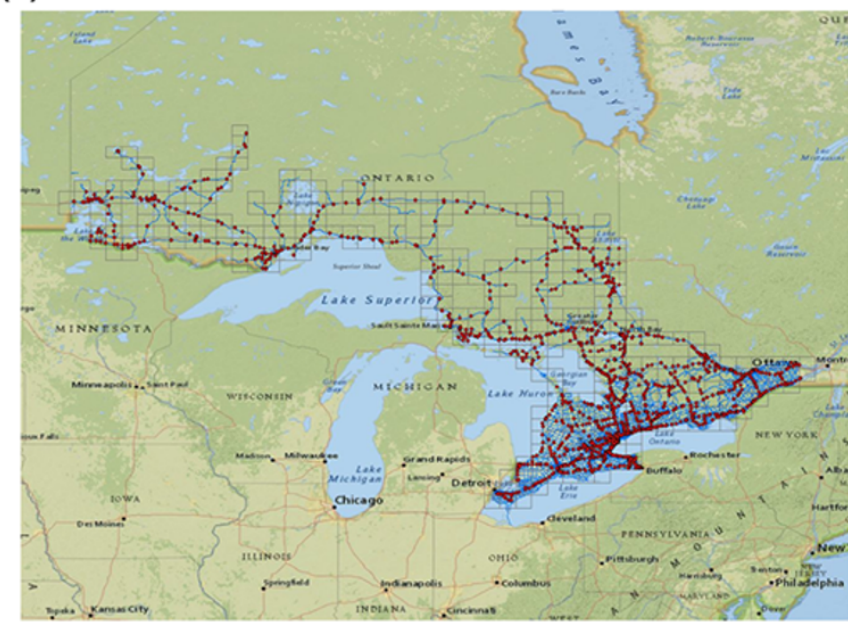

(c)

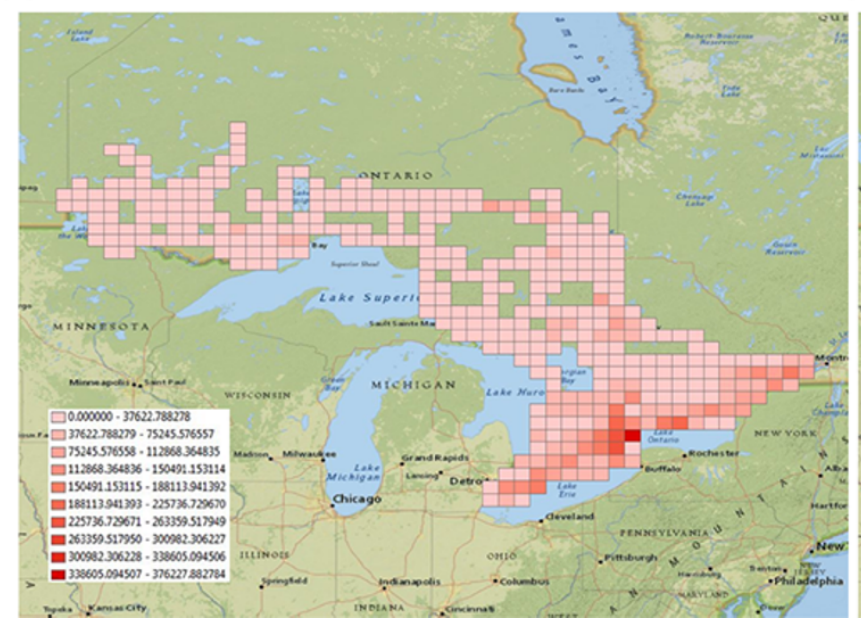

(b)

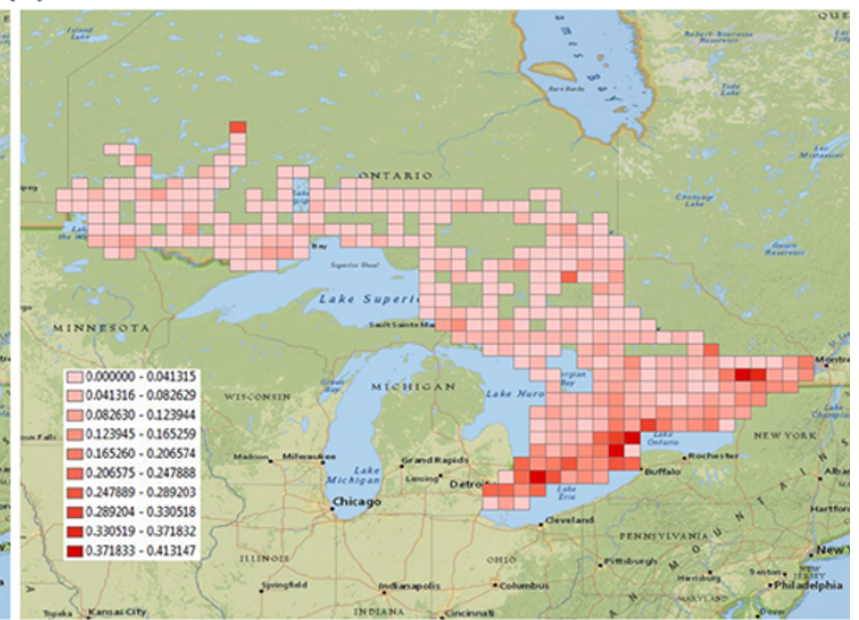

(d)

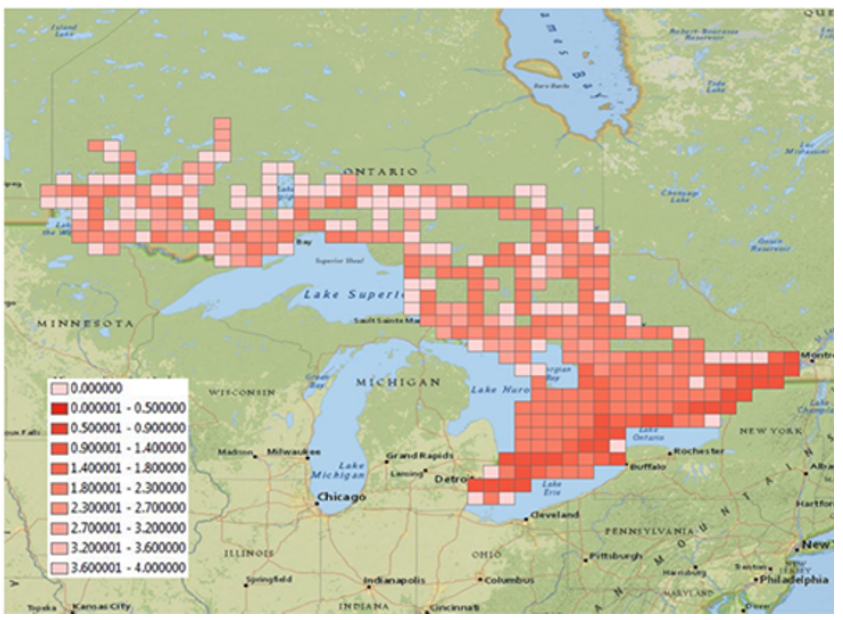

Fig. 5 a LHRS, b WAR, c WADT, and d HT

fact that VST and MST are positively correlated indicates that when MST is lower, its variability would be higher. It is interesting to see some degree of correlation between WADT and MST. This is probably because the low MST values are present mostly in the northern regions whereas a majority of high WADT values appear in the southern regions. Hence, two weather factors of VST and SWE, and two traffic factors of WAR and HT are considered for the evaluation.

\subsubsection{Alternative 1: weather factors combined}

Two pre-defined weather factors namely VST and SWE are used to evaluate the current RWIS network in the province of Ontario. VST values in each cell have been added to the corresponding cell of SWE. Note that both factors are normalized with a range between 0 and 1 to eliminate any potential bias. Figure 6 describes the result of the combined location selection criteria. It also shows the current
Table 1 Spearman's rank correlation coefficients

\begin{tabular}{lllllcc}
\hline & VST & MST & SWE & HT & WAR & WADT \\
\hline VST & 1 & 0.643638 & 0.194449 & -0.42406 & -0.4318 & -0.43333 \\
MST & - & 1 & 0.298177 & -0.46556 & -0.50343 & -0.54187 \\
SWE & - & - & 1 & -0.12457 & -0.16166 & -0.1729 \\
HT & - & - & - & 1 & 0.39289 & 0.351165 \\
WAR & - & - & - & - & 1 & 0.555482 \\
WADT & - & - & - & - & - & 1 \\
\hline
\end{tabular}


Ontario RWIS stations that have been superimposed on the map. Highlighted cells represent the top 140 cells that are recommended as potential RWIS station locations.

As can be seen in the figure, a map generated by combining two weather factors suggests a potential RWIS site to be in the middle to upper part of the region, where VST and SWE are also found to be significant in those regions. A percent of matching is found to be $24 \%$ by having 33 cells matched with the actual locations of the existing RWIS stations. Notice that there are many cells in the central regions (i.e., highlighted cells) where no RWIS stations are there to monitor the highly varying weather conditions with historically heavy snowfall events. From this analysis, it can be stated that the current RWIS location setting is less susceptible to capturing the variability of weather conditions.

\subsubsection{Alternative 2: traffic factors combined}

A second alternative contains the two predetermined traffic-related factors namely WAR and HT. Figure 7 illustrates the proposed 140 locations of RWIS stations when considering the traffic factors only. Notice that the map now focuses more on the areas where high accident rates/ highway class exist. This alternative suggests that almost all southern parts of the province should have RWIS stations installed while most parts in the northern region are left uncovered. Ninety-three of the 140 existing RWIS stations (66\%) would be located at the same sites based on this alternative. Such a high matching rate should not be viewed as an indication that this location criterion is the best; instead, it should rather be considered as an indication that these factors are most heavily weighted in Ontario's current RWIS location practice.

\subsubsection{Alternative 3: weather and traffic factors combined}

A third alternative is proposed by combining both weather and traffic factors to balance out the deficiencies and limitations of alternatives 1 and 2. Figure 8 shows the proposed 140 locations where RWIS stations are recommended to be sited when considering all four factors previously explained. A visual inspection of the result shows that the identified cells are well distributed over the entire province. The percent of matching for alternative 3 is found to be smaller (i.e., $57 \%$ ) than that of alternative 2. However it is worthwhile to emphasize that such a number is based on the evaluation with respect to the current locations of Ontario RWIS stations, and thus does not provide an absolute measure of the performance. While the current RWIS setting is able to provide a reasonably good coverage on both traffic and weather factors, the large difference between the two suggests that the RWIS stations may not have been located optimally.

\section{Conclusions}

In this study, a general framework for choosing the location of a RWIS station at a regional level has been developed. Several location selection criteria are proposed on the basis of scientific and/or rational justifications that they would, to a certain extent, help us delineate good RWIS locations.
Fig. 6 Alternative 1 with the two weather factors combined

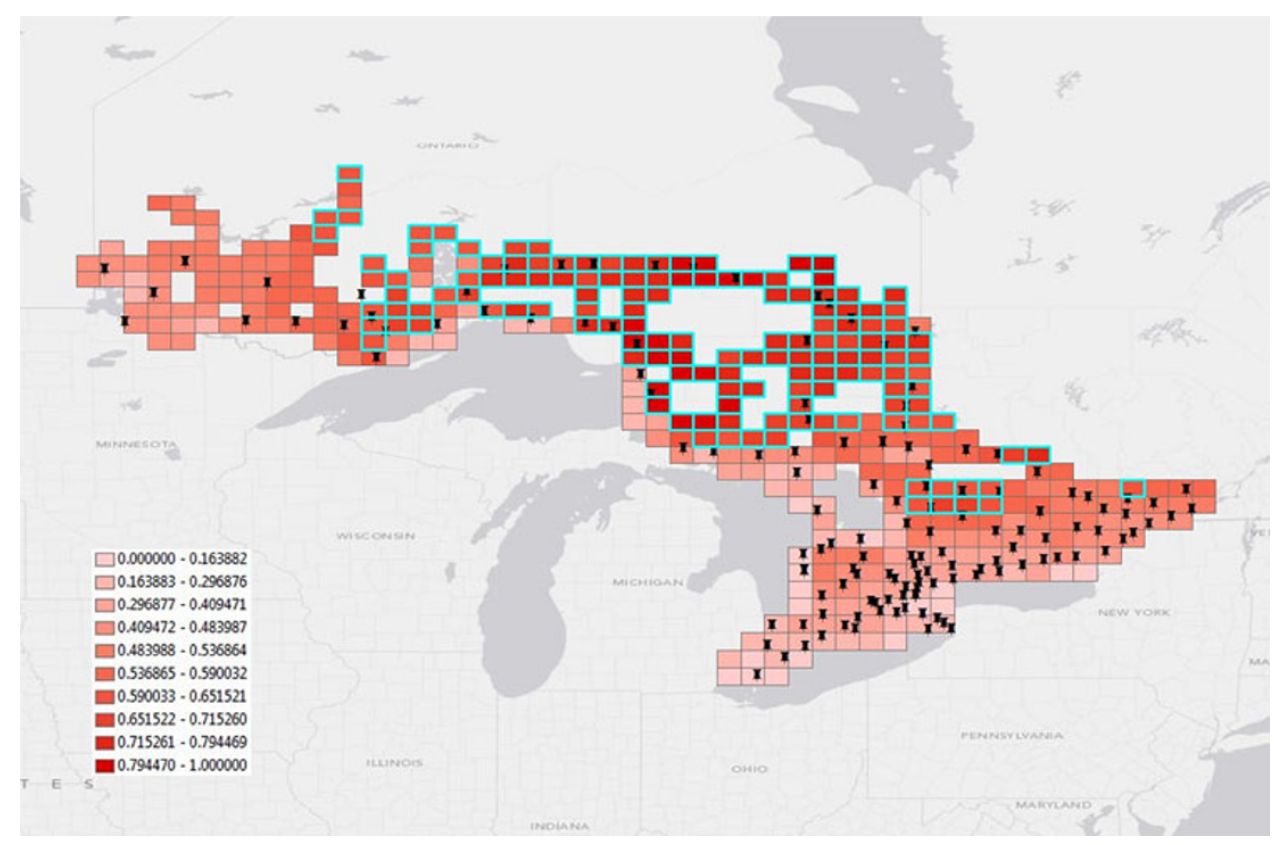



two traffic factors combined
Fig. 7 Alternative 2 with the

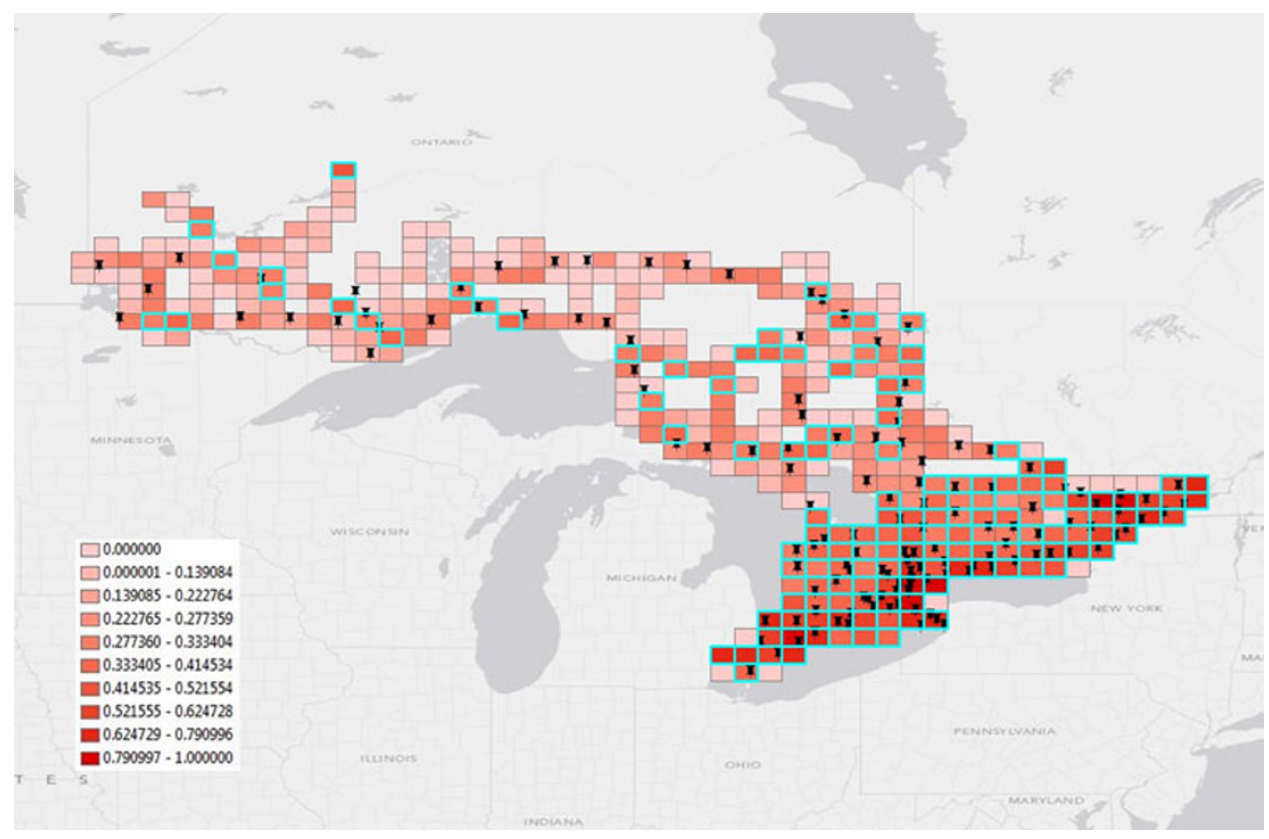

Fig. 8 Alternative 3 with all four factors combined

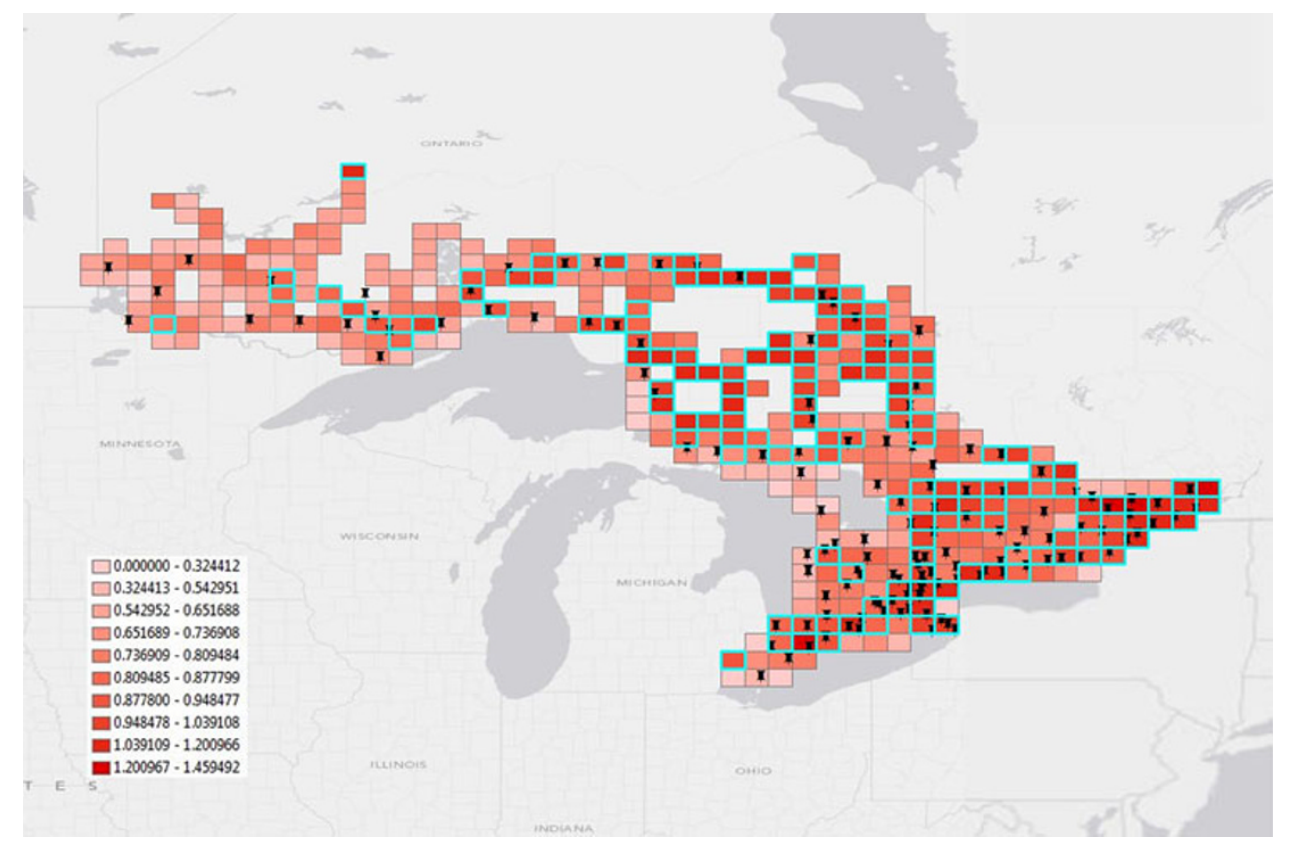

Three weather factors considered include VST, MST, and snow water equivalent (SWE), of which VST and MST are modeled by taking into account the several locational attributes. WADT, WAR, and HT are also considered under the rationale that serving a higher number of traveling publics would provide more benefits. After confirming the multicollinearity between the considered variables, a total of three location selection alternatives have been developed: alternatives 1 and 2 take into account two weather factors, VST and SWE; and two traffic factors, WAR and HT, respectively. Alternative 3 is a combination of alternatives 1 and 2. These alternatives are used to evaluate the current Ontario RWIS network. A percent of matching is utilized as an evaluation metric for benchmarking the current location setting. The findings have revealed that the alternative 1 is more focused on the northern region comprising of highly varying weather conditions with $24 \%$ matching rate, while the alternative 2 is more focused on the southern region with heavy traffic loads with $66 \%$ matching rate. The high matching rate of the alternative 2 indicates that the current RWIS network has been set up in such way that it predominantly considers 
the need of covering the road network. The alternative 3 with $57 \%$ matching rate seems to balance the limitations of the first two alternatives by showing the potential candidate RWIS locations across the whole province. It is unknown how much of weight needs to be put on each of the criterion discussed here to have an optimized RWIS network, but it is clear that the proposed framework is easy to apply when planning a RWIS network expansion by introducing different weights to individual criteria based on their importance.

This research can be extended in several directions. First, VST and MST models can be improved by utilizing a geostatistical interpolation technique such as kriging. Several studies have found that kriging would provide a better estimation than regression, especially when variables are spatially dependent on each other $[15,16]$. Second, the use of LHRS needs to be improved since the cells that do not have a reference point will have no data. Third, methodical guidelines need to be established for determining a number of RWIS stations to be allocated within a cell. This is particularly important for DOTs wanting to install more than one RWIS station within, for instance, as used herein minimum spatial unit of $50 \mathrm{~km}^{2}$ to enhance and extend their monitoring capability and spatial coverage. Finally, it is of necessity to establish quantitative models for estimating the benefit of using information from RWIS stations, with which a cost-benefit based RWIS location optimization model can be developed.

Acknowledgments The authors wish to thank Max Perchanok, Heather McClintock, Mary Anne Grepsma, and Bill Harrett at the Ontario Ministry of Transportation. This research was funded by the Aurora Program, and was partially funded by National Sciences and Engineering Research Council of Canada (NSERC) and Ontario Ministry of Transportation (MTO).

Open Access This article is distributed under the terms of the Creative Commons Attribution License which permits any use, distribution, and reproduction in any medium, provided the original author(s) and the source are credited.

\section{References}

1. Garrett JK, Boyce B, Krechmer D (2008) Implementation and evaluation of RWIS ESS siting guide. Federal Highway Administration Road Weather Management Program

2. Buchanan F, Gwartz SE (2005) Road weather information systems at the ministry of transportation. Transportation Association of Canada, Ontario

3. Canadian Strategic Highway Research Program (2000) AntiIcing and RWIS Technology in Canada

4. Ye Z, Strong S, Fay L et al (2009) Cost benefits of weather information for winter road maintenance. Final report, Iowa Department of Transportation

5. Boselly SE (2001) Benefit/cost study of RWIS and anti-icing technologies. National Cooperative Highway Research Program, TRB

6. Eriksson M, Norrman J (2001) Analysis of station locations in a road weather information system. Meteorol Appl 448(8):437-448

7. World Weather Watch (2009) Climatological assessment for the purposes of siting road sensors. Final Report

8. Mackinnon D, Lo A (2009) Alberta Transportation Road Weather Information System (RWIS) expansion study. Alberta Transportation

9. Greening K, Johns D, Bridge P et al (2012) A study to determine the effects of employing a well maintained RWIS network on accident rates on major highways in the US state of Idaho. Standing International Road Weather Commission, Helsinki

10. Ahrens CD (2009) Meteorology today: an introduction to weather, climate, and the environment. Brooks/Cole, Cengage Learning, Belmont

11. Thornton PE, Thornton MM, Mayer BW et al (2012) Daymet: annual surface weather on a $1 \mathrm{~km}$ grid for North America, Acquired on 16 December 2012 from Oak Ridge National Laboratory Distributed Active Archive Center, Oak Ridge. doi: 10.3334/ORNLDAAC/Daymet_V2

12. Thornton PE, Hasenauer H, White MA (2000) Simultaneous estimation of daily solar radiation and humidity from observed temperature and precipitation: an application over complex terrain in Austria. Agric For Meteorol 104(4):255-271

13. Baker W, Blessing W (1974) Highway linear reference methods, synthesis of highway practice 21, National Cooperative Highway Research Program. National Academy Press, Washington

14. Steel GDR, Torrie JH (1980) Principles and procedures of statistics: a biometrical approach, 2nd edn. McGraw-Hill, New York

15. Hengl T, Heuvelink GBM, Stein A (2003) Comparison of kriging with external drift and regression-kriging. International Institute for Geo-Information Science and Earth Observation (ITC), Enschede

16. Mesquita S, Sousa AJ (2009) Bioclimatic mapping using geostatistical approaches: application to mainland Portugal. Int J Climatol 29(14):2156-2170 